\title{
ILW EQUATION FOR THE HODGE INTEGRALS REVISITED
}

\author{
ALEXANDR BURYAK
}

\begin{abstract}
In a previous paper we proved that after a simple transformation the generating series of the linear Hodge integrals on the moduli space of stable curves satisfies the hierarchy of the Intermediate Long Wave equation. In this paper we present a much shorter proof of this fact. Our new proof is based on an explicit formula for the one-point linear Hodge integrals that was found independently by Faber, Pandharipande and Ekedahl, Lando, Shapiro, Vainshtein.
\end{abstract}

\section{INTRODUCTION}

Consider the moduli space $\overline{\mathcal{M}}_{g, n}$ of stable complex algebraic curves of genus $g$ with $n$ marked points. The class $\psi_{i} \in H^{2}\left(\overline{\mathcal{M}}_{g, n} ; \mathbb{C}\right)$ is defined as the first Chern class of the line bundle over $\overline{\mathcal{M}}_{g, n}$ formed by the cotangent lines at the $i$-th marked point. Denote by $\lambda_{j} \in H^{2 j}\left(\overline{\mathcal{M}}_{g, n} ; \mathbb{C}\right)$ the $j$-th Chern class of the rank $g$ Hodge vector bundle over $\overline{\mathcal{M}}_{g, n}$ whose fibers over smooth curves are the spaces of holomorphic one-forms. In [Bur15] we studied the following integrals:

$$
\left\langle\lambda_{j} \tau_{d_{1}} \tau_{d_{2}} \ldots \tau_{d_{n}}\right\rangle_{g}:=\int_{\overline{\mathcal{M}}_{g, n}} \lambda_{j} \psi_{1}^{d_{1}} \psi_{2}^{d_{2}} \ldots \psi_{n}^{d_{n}} .
$$

In the unstable cases $2 g-2+n \leq 0$ we define the bracket to be equal to zero. Introduce variables $\hbar, \varepsilon$ and $t_{0}, t_{1}, t_{2}, \ldots$ and consider the generating series

$$
F^{\text {Hodge }}\left(t_{0}, t_{1}, \ldots ; \hbar, \varepsilon\right):=\sum_{n \geq 0} \sum_{0 \leq j \leq g} \frac{\hbar^{g} \varepsilon^{j}}{n !} \sum_{d_{1}, \ldots, d_{n} \geq 0}\left\langle\lambda_{j} \tau_{d_{1}} \ldots \tau_{d_{n}}\right\rangle_{g} t_{d_{1}} \ldots t_{d_{n}} .
$$

Let

$$
\widetilde{F}^{\text {Hodge }}:=F^{\text {Hodge }}+\sum_{g \geq 1} \frac{(-1)^{g}}{2^{2 g}(2 g+1) !} \hbar^{g} \varepsilon^{g} \frac{\partial^{2 g} F^{\text {Hodge }}}{\partial t_{0}^{2 g}} .
$$

In [Bur15] we proved the following theorem.

Theorem 1.1. The second derivative $u=\frac{\partial^{2} \widetilde{F}^{\text {Hodge }}}{\partial t_{0}^{2}}$ satisfies the hierarchy of the Intermediate Long Wave equation:

$$
\begin{aligned}
& \frac{\partial u}{\partial t_{1}}=u u_{x}+\sum_{g \geq 1} \hbar^{g} \varepsilon^{g-1} \frac{\left|B_{2 g}\right|}{(2 g) !} u_{2 g+1}, \\
& \frac{\partial u}{\partial t_{2}}=\frac{1}{2} u^{2} u_{x}+\sum_{g \geq 1} \frac{\left|B_{2 g}\right|}{(2 g) !} \hbar^{g} \frac{\varepsilon^{g-1}}{4}\left(2\left(u u_{2 g}\right)_{x}+\partial_{x}^{2 g+1}\left(u^{2}\right)\right)+\sum_{g \geq 2} \frac{\left|B_{2 g}\right|}{(2 g) !} \hbar^{g} \varepsilon^{g-2}(g+1) u_{2 g+1},
\end{aligned}
$$

Here we identify $x$ with $t_{0}, B_{2 g}$ stand for the Bernoulli numbers: $B_{2}=\frac{1}{6}, B_{4}=-\frac{1}{30}, \ldots$; and we denote by $u_{i}$ the derivative $\partial_{x}^{i} u$.

Let us say a few words about the hierarchy of the Intermediate Long Wave (ILW) equation (see e.g. [SAK79]), since it is less known in the mathematical literature than, for example, the KdV hierarchy. The ILW equation is actually the first equation (1.2) of the hierarchy. It can 
be written in a hamiltonian form:

$$
\begin{aligned}
& \frac{\partial u}{\partial t_{1}}=\partial_{x} \frac{\delta \bar{h}_{1}^{\mathrm{ILW}}}{\delta u}, \quad \text { where } \\
& \bar{h}_{1}^{\mathrm{ILW}}=\int\left(\frac{u^{3}}{6}+\sum_{g \geq 1} \hbar^{g} \varepsilon^{g-1} \frac{\left|B_{2 g}\right|}{2(2 g) !} u u_{2 g}\right) d x .
\end{aligned}
$$

The whole ILW hierarchy is also hamiltonian. The higher Hamiltonians $\bar{h}_{i}^{\mathrm{ILW}}, i \geq 2$, have the form

$$
\bar{h}_{i}^{\mathrm{ILW}}=\int\left(\frac{u^{i+2}}{(i+2) !}+O(\hbar)\right) d x,
$$

and, by [Bur15, Lemma 2.4], are uniquely determined by the commutation relation

$$
\left\{\bar{h}_{i}^{\mathrm{ILW}}, \bar{h}_{1}^{\mathrm{ILW}}\right\}_{\partial_{x}}:=\int \frac{\delta \bar{h}_{i}^{\mathrm{ILW}}}{\delta u} \partial_{x} \frac{\delta \bar{h}_{1}^{\mathrm{ILW}}}{\delta u} d x=0 .
$$

An explicit construction of the higher Hamiltonians can be found in [SAK79].

Remark 1.2. We must say that our definition of the ILW hierarchy is slightly different from the one that is more common in the physics literature (see e.g. SAK79]). First of all, the ILW equation from SSAK79] is different from ours by several rescalings and, second, the higher Hamiltonians from [SAK79] are related to ours by some triangular transformation. We discuss the precise relation in Bur15.

Remark 1.3. In [Bur15] the hierarchy (1.2) is called the deformed KdV hierarchy. This happened because at the moment when the result of [Bur15] was obtained we didn't know that this hierarchy already appeared in a literature. We are very grateful to S. Ferapontov and D. Novikov for recognizing the ILW equation in the first equation of (1.2) after the author's talk on the conference in Trieste (Hamiltonian PDEs, Frobenius manifolds and Deligne-Mumford moduli spaces, September 2013).

Our approach in [Bur15] is based on the Dubrovin and Zhang theory generalized in [BPS12a] (see also [BPS12b]). Let us briefly recall it. Suppose we have a semisimple cohomological field theory (not necessarily homogeneous) with a metric $\eta$ and a potential $F$. Then in BPS12a. it is presented a construction of a hamiltonian hierarchy of PDEs with a solution given by the second derivatives

$$
w^{\alpha}=\eta^{\alpha \gamma} \frac{\partial^{2} F}{\partial t_{0}^{1} \partial t_{0}^{\gamma}} .
$$

Consider the cohomological field theory formed by the full Chern class of the Hodge bundle:

$$
1+\varepsilon \lambda_{1}+\varepsilon \lambda_{2}+\ldots+\varepsilon^{g} \lambda_{g} \in H^{*}\left(\overline{\mathcal{M}}_{g, n} ; \mathbb{C}\right) .
$$

In Bur15] we showed that Theorem 1.1 is a corollary of the following result.

Theorem 1.4. Consider the Dubrovin-Zhang hierarchy associated to the cohomological field theory (1.3). Then the Miura transformation

$$
w \mapsto u=w+\sum_{g \geq 1} \frac{(-1)^{g}}{2^{2 g}(2 g+1) !} \hbar^{g} \varepsilon^{g} w_{2 g}
$$

transforms this hierarchy to the ILW hierarchy.

The proof of Theorem 1.4 in Bur15] consists of two steps:

(1) Note that the class (1.3) lies in cohomological degrees not greater than $2 g$. Remarkably, this simple fact together with the polynomiality property of the Dubrovin-Zhang hierarchy, proved in [BPS12a] (see also [BPS12b]), and an explicit formula for the $\lambda_{g^{-}}$ integrals, proved in [FP03, imply that the Miura transformation (1.4) transforms the 
first hamiltonian operator of the Dubrovin-Zhang hierarchy to the operator $\partial_{x}$ and the first Hamiltonian to a local functional of the form

$$
\int\left(\frac{u^{3}}{6}+\sum_{g \geq 1} \hbar^{g} \varepsilon^{g-1} C_{g} u u_{2 g}\right) d x
$$

where $C_{g}$ are some constants.

(2) The second step consists of the computation of the constants $C_{g}$. In [Bur15] we observed that an existence of a local functional of the form

$$
\int\left(\frac{u^{4}}{24}+O(\hbar)\right) d x
$$

that commutes with (1.5), gives strong constraints for the coefficients $C_{g}$. This idea together with the explicit computation of the coefficients $C_{1}$ and $C_{2}$ allowed us to prove that $C_{g}=\frac{\left|B_{2 g}\right|}{2(2 g) !}$.

While the first step is a direct computation (see [Bur15, Section 5]) based on the construction of the Dubrovin-Zhang hierarchy from BPS12a, the second step in Bur15 involves quite technical computations (see [Bur15, Sections 6,7 and Appendix A and B]).

In the present paper we do the second step in a much simpler way. We give a short computation of the constants $C_{g}$ using the explicit formula for the one-point linear Hodge integrals that was found independently in [FP00] and [ELSV99].

For a reader who is not familiar with details of the Dubrovin-Zhang theory we would like to make the following summary. In [Bur15, Section 5] we proved that the second derivative $u=\frac{\partial^{2} \widetilde{F}^{\text {Hodge }}}{\partial t_{0}^{2}}$ satisfies an equation of the form

$$
\frac{\partial u}{\partial t_{1}}=u u_{x}+\sum_{g \geq 1} \hbar^{g} \varepsilon^{g-1} 2 C_{g} u_{2 g+1},
$$

for some constants $C_{g}$. In Section 2 we present a short computation of the constants $C_{g}$. This gives another proof of Theorem 1.4. The computation in Section 2 doesn't use any material from [Bur15. So it is possible to read Section 2 even without looking inside [Bur15].

Remark 1.5. Our argument from Section 2 can be easily applied in the opposite direction. It shows that the formula from [FP00] and [ELSV99] for the generating series of the one-point linear Hodge integrals (formula (2.2) ) follows from Theorem 1.1.

1.1. Acknowledgements. We would like to thank R. Pandharipande and P. Rossi for useful discussions.

The author was supported by grant ERC-2012-AdG-320368-MCSK in the group of R. Pandharipande at ETH Zurich, by grants RFFI 13-01-00755 and NSh-4850.2012.1.

\section{Computation of the constants $C_{g}$}

We have the equation

$$
\frac{\partial u}{\partial t_{1}}=u u_{x}+\sum_{g \geq 1} \hbar^{g} \varepsilon^{g-1} 2 C_{g} u_{2 g+1},
$$

where $u=\frac{\partial^{2} \widetilde{F}_{\text {Hodge }}}{\partial t_{0}^{2}}$ and $C_{g}$ are some constants. Let us prove that $C_{g}=\frac{\left|B_{2 g}\right|}{2(2 g) !}$.

Consider formal variables $t$ and $k$. The following formula was obtained independently in [FP00] and [ELSV99]:

$$
1+\sum_{g \geq 1} \sum_{i=0}^{g} t^{2 g} k^{i}\left\langle\lambda_{g-i} \tau_{2 g-2+i}\right\rangle_{g}=\left(\frac{t / 2}{\sin (t / 2)}\right)^{k+1} .
$$


Let us slightly reformulate this result. We have the string equation

$$
\frac{\partial F^{\text {Hodge }}}{\partial t_{0}}=\sum_{n \geq 0} t_{n+1} \frac{\partial F^{\text {Hodge }}}{\partial t_{n}}+\frac{t_{0}^{2}}{2}+\frac{\hbar \varepsilon}{24} .
$$

Note also that the bracket (1.1) vanishes unless the dimension constraint

$$
3 g-3+n=j+\sum_{i=1}^{n} d_{i}
$$

is satisfied. Let

$$
S(\hbar, \varepsilon):=\sum_{0 \leq j \leq g}\left\langle\lambda_{j} \tau_{0}^{2} \tau_{3 g-j}\right\rangle_{g} \hbar^{g} \varepsilon^{j} .
$$

By the string equation (2.3), formula (2.2) is equivalent to the following equation:

$$
S(\hbar, \varepsilon)=\left(\frac{\sqrt{\hbar \varepsilon} / 2}{\sin (\sqrt{\hbar \varepsilon} / 2)}\right)^{1+\frac{1}{\varepsilon}}
$$

Let

$$
\left\langle\lambda_{j} \tau_{d_{1}} \tau_{d_{2}} \ldots \tau_{d_{n}}\right\rangle_{g}^{\sim}:=\left.\operatorname{Coef}_{\hbar^{g} \varepsilon^{j}} \frac{\partial^{n} \widetilde{F}^{\text {Hodge }}}{\partial t_{d_{1}} \partial t_{d_{2}} \ldots \partial t_{d_{n}}}\right|_{t_{*}=0}
$$

It is easy to see that, similarly to the bracket (1.1), the new bracket (2.6) vanishes unless the dimension constraint (2.4) is satisfied. It is also clear that the new bracket is zero when $j>g$. Let

$$
\widetilde{S}(\hbar, \varepsilon):=\sum_{0 \leq j \leq g}\left\langle\lambda_{j} \tau_{0}^{2} \tau_{3 g-j}\right\rangle_{g}^{\sim} \hbar^{g} \varepsilon^{j}
$$

Using the string equation (2.3) we get

$$
\begin{aligned}
\widetilde{S}(\hbar, \varepsilon) & =\left(1+\sum_{g \geq 1} \frac{(-1)^{g}}{2^{2 g}(2 g+1) !} \hbar^{g} \varepsilon^{g}\right) S(\hbar, \varepsilon)=\frac{\sin (\sqrt{\hbar \varepsilon} / 2)}{\sqrt{\hbar \varepsilon} / 2} S(\hbar, \varepsilon) \stackrel{\text { by } \underline{\underline{(2.5)}}}{=} \\
& =\left(\frac{\sqrt{\hbar \varepsilon} / 2}{\sin (\sqrt{\hbar \varepsilon} / 2)}\right)^{1 / \varepsilon}=\exp \left(\frac{1}{\varepsilon} \log \left(\frac{\sqrt{\hbar \varepsilon} / 2}{\sin (\sqrt{\hbar \varepsilon} / 2)}\right)\right) .
\end{aligned}
$$

Note that

$$
\log \left(\frac{z / 2}{\sin (z / 2)}\right)=\sum_{g \geq 1} \frac{\left|B_{2 g}\right|}{2 g(2 g) !} z^{2 g}
$$

Therefore,

$$
\widetilde{S}(\hbar, \varepsilon)=\exp \left(\sum_{g \geq 1} \hbar^{g} \varepsilon^{g-1} \frac{\left|B_{2 g}\right|}{2 g(2 g) !}\right) .
$$

Let us now compute $\widetilde{S}(\hbar, \varepsilon)$ using equation (2.1). The string equation (2.3) for $F^{\text {Hodge }}$ implies the string equation for $\widetilde{F}^{\text {Hodge }}$ :

$$
\frac{\partial \widetilde{F}^{\text {Hodge }}}{\partial t_{0}}=\sum_{n \geq 0} t_{n+1} \frac{\partial \widetilde{F}^{\text {Hodge }}}{\partial t_{n}}+\frac{t_{0}^{2}}{2} .
$$


It gives the property $\left.u_{d}\right|_{t_{*}=0}=\delta_{d, 1}$. Therefore, we can compute the linear term in $t_{i}$ 's of the expression on the right-hand side of equation (2.1) in the following way:

$$
\begin{aligned}
& u u_{x}+\sum_{g \geq 1} \hbar^{g} \varepsilon^{g-1} 2 C_{g} u_{2 g+1}= \\
= & \sum_{0 \leq j \leq g} \hbar^{g} \varepsilon^{j}\left\langle\lambda_{j} \tau_{0}^{2} \tau_{3 g-j}\right\rangle_{g}^{\sim} t_{3 g-j}+\sum_{g \geq 1} 2 C_{g} \sum_{0 \leq j \leq h} \hbar^{g+h} \varepsilon^{g+j-1}\left\langle\lambda_{j} \tau_{0}^{2} \tau_{3 h-j}\right\rangle_{h}^{\sim} t_{3(g+h)-(g+j-1)}+O\left(t^{2}\right) .
\end{aligned}
$$

Let us look on the left-hand side of equation (2.1). We have the dilaton equation for the potential $F^{\text {Hodge: }}$

$$
\frac{\partial F^{\text {Hodge }}}{\partial t_{1}}=\left(\sum_{n \geq 0} t_{n} \frac{\partial}{\partial t_{n}}+2 \hbar \frac{\partial}{\partial \hbar}-2\right) F^{\text {Hodge }}+\frac{\hbar}{24} .
$$

It implies the dilaton equation for the transformed potential $\widetilde{F}^{\text {Hodge }}$ :

$$
\frac{\partial \widetilde{F}^{\text {Hodge }}}{\partial t_{1}}=\left(\sum_{n \geq 0} t_{n} \frac{\partial}{\partial t_{n}}+2 \hbar \frac{\partial}{\partial \hbar}-2\right) \widetilde{F}^{\text {Hodge }}+\frac{\hbar}{24} .
$$

This allows us to compute the linear term in $t_{i}$ 's of the expression on the left-hand side of (2.1) in the following way:

$$
\frac{\partial u}{\partial t_{1}}=\sum_{0 \leq j \leq g} \hbar^{g} \varepsilon^{j}(2 g+1)\left\langle\lambda_{j} \tau_{0}^{2} \tau_{3 g-j}\right\rangle_{g}^{\sim} t_{3 g-j}+O\left(t^{2}\right) .
$$

Equating (2.8) and (2.9) $)$, we get

$$
\sum_{0 \leq j \leq g} \hbar^{g} \varepsilon^{j}(2 g)\left\langle\lambda_{j} \tau_{0}^{2} \tau_{3 g-j}\right\rangle_{g}^{\sim} t_{3 g-j}=\sum_{g \geq 1} 2 C_{g} \sum_{0 \leq j \leq h} \hbar^{g+h} \varepsilon^{g+j-1}\left\langle\lambda_{j} \tau_{0}^{2} \tau_{3 h-j}\right\rangle_{h}^{\sim} t_{3(g+h)-(g+j-1)} .
$$

We can rewrite this equation as a differential equation for $\widetilde{S}(\hbar, \varepsilon)$ :

$$
\frac{\partial}{\partial \hbar} \widetilde{S}(\hbar, \varepsilon)=\left(\sum_{g \geq 1} C_{g}(\hbar \varepsilon)^{g-1}\right) \widetilde{S}(\hbar, \varepsilon) .
$$

Therefore,

$$
\widetilde{S}(\hbar, \varepsilon)=\exp \left(\sum_{g \geq 1} \frac{C_{g}}{g} \hbar^{g} \varepsilon^{g-1}\right) .
$$

Comparing this equation with equation (2.7), we get

$$
C_{g}=\frac{\left|B_{2 g}\right|}{2(2 g) !} \text {. }
$$

This completes our proof.

\section{REFERENCES}

[Bur15] A. Buryak, Dubrovin-Zhang hierarchy for the Hodge integrals, Communication in Number Theory and Physics 9 (2015), no. 2, 239-271. arXiv:1308.5716.

[BPS12a] A. Buryak, H. Posthuma, S. Shadrin, On deformations of quasi-Miura transformations and the Dubrovin-Zhang bracket, Journal of Geometry and Physics 62 (2012), no. 7, 1639-1651.

[BPS12b] A. Buryak, H. Posthuma, S. Shadrin, A polynomial bracket for the Dubrovin-Zhang hierarchies, Journal of Differential Geometry 92 (2012), no. 1, 153-185.

[DZ05] B. A. Dubrovin, Y. Zhang, Normal forms of hierarchies of integrable PDEs, Frobenius manifolds and Gromov-Witten invariants, a new 2005 version of arXiv:math/0108160v1, 295 pp.

[ELSV99] T. Ekedahl, S. Lando, M. Shapiro, A. Vainshtein, On Hurwitz numbers and Hodge integrals, Comptes Rendus de l'Académie des Sciences. Série I. Mathématique 328 (1999), no. 12, 1175-1180. 
[FP00] C. Faber, R. Pandharipande, Hodge integrals and Gromov-Witten theory, Inventiones Mathematicae 139 (2000), no. 1, 173-199.

[FP03] C. Faber, R. Pandharipande, Hodge integrals, partition matrices, and the $\lambda_{g}$-conjecture, Annals of Mathematics 157 (2003), no. 1, 97-124.

[SAK79] J. Satsuma, M. J. Ablowitz, Y. Kodama, On an internal wave equation describing a stratified fluid with finite depth, Physics Letters A 73 (1979), no. 4, 283-286.

Department of Mathematics, ETH Zurich, Rämistrasse 101 8092, Zurich, SwitzerLAnd

E-mail address: buryaksh@gmail.com 